The activation energy of the process is, of course, reduced by more positive anode potentials (much as in a simple charge-transfer process) and the probability of formation of the high-energy transitional complexes increases with rise of temperature, in the usual way.

\section{T. P. HOAR}

Department of Metallurgy,

University of Cambridge.

Received November 28, 1967.

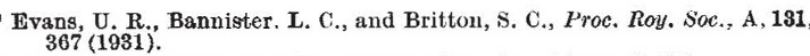

'Hoar, T. P., and Evans, U. R., J. Electrochem. Soc., 99, 212 (1952).

${ }^{3}$ Kolotyrkin, Ya. M., Corrosion, 18, 263t (1963).

4 Leckie, H. P., and Uhlig, H. H., J. Electrochem. Soc., 113, 1262 (1966).

' Hoar, T. P., Mears, D. C., and Rothwell, G. P., Corros. Sci.. 5, 279 (1985).

"Hoar, T. P., Corros. Sci., 7, 341 (1967).

\section{CHEMISTRY}

\section{Microwave Spectra and Structures of Isothiazole, I,2,4-Oxadiazole and Imidazole}

ROTATIONAL spectroscopy has proved a powerful method of studying the structures of heterocyclic molecules ${ }^{1-6}$. We have extended work of this type to molecules of isothiazole, 1,2,4-oxadiazole and imidazole.

Spectral assignments followed established lines, absorptions being observed for $J$ values between 0 and 18 and in general for both $A$ and $B$ components of the dipole moments. To observe the spectra of imidazole, the sample and absorption cell were maintained at about $50^{\circ} \mathrm{C}$ to provide sufficient vapour pressure. Rotational constants were derived as follows (in Mc/s)

$\begin{array}{lccc} & A & B & C \\ \text { Isothiazole } & 8,275 \cdot 52 \pm 0 \cdot 1 & 5,846 \cdot 24 \pm 0 \cdot 05 & 3,424 \cdot 21 \pm 0 \cdot 05 \\ \text { 1,2,4-Oxadiazole } & 10,337 \cdot 37 \pm 0 \cdot 2 & 10,092 \cdot 66 \pm 0 \cdot 1 & 5,103 \cdot 33 \pm 0 \cdot 1 \\ \text { Imidazole } & 9,725 \cdot 06 \pm 0 \cdot 2 & 9,373 \cdot 92 \pm 0 \cdot 2 & 4,772 \cdot 02 \pm 0 \cdot 2 \\ \begin{array}{c}\text { N-Deutero- } \\ \text { imidazole }\end{array} & 9,668 \cdot 31 \pm 0 \cdot 2 & 8,699 \cdot 39 \pm 0 \cdot 2 & 4,578 \cdot 43 \pm 0 \cdot 2\end{array}$

The moments of inertia show that all the molecules are planar, the small inertial defects being close to those found for furan ${ }^{1}$, pyrrole ${ }^{2}$, isomeric oxadiazoles ${ }^{3,4}$, thia$z^{2} e^{5}$ and pyrazole ${ }^{8}$. The moments of inertia of imidazole are somewhat larger than for the molecular dimensions found by X-ray diffraction in the crystalline state $\theta^{7}$. This lack of agreement is not unexpected in view of the strong intermolecular interactions, and the short $\mathrm{CH}$ distances, reported in the solid. The effect, on the constants, of deuteration of the imino group allows the imino hydrogen to be located in the inertial axis frame; for any acceptable model this means that the $A$ axis in imidazole makes an angle of about $28^{\circ}$ with the NH bond. A study of numerous models with reasonable structure parameters suggests that this axis intersects the NC bond joining the 1 and 2 positions.

Nuclear quadrupole hyperfine structure was resolvable in all these spectra. For isothiazole, the coupling constants in the inertial axes are (in $\mathrm{Mc} / \mathrm{s}$ ):

$$
\chi_{a \mathrm{a}}=1.05 \pm 0 \cdot 2 \quad \chi_{\mathrm{bb}}=-2.42 \pm 0 \cdot 1 \quad \chi_{\mathrm{cc}}=1.37 \pm 0.3
$$

These are acceptable values in view of the findings for similar cases and the approximately known directions of the $A$ and $B$ axes in the molecule. For 1,2,4-oxadiazole, well resolved splittings, caused by the two non-equivalent nitrogen nuclei, occur. Although the coupling constants for the different nuclei may clearly be of comparable magnitude, an analysis, in terms of individual coupling constants, should be possible and is proceeding. We expect that the hyperfine structures for imidazole will be similarly analysed with anticipated improvements in resolution.

Analysis of Stark effects for a number of transitions of each substance leads to the following preliminary values of the dipole moment components (in $D$ )

$\begin{array}{lccr} & \mu_{\mathrm{a}} & \mu \mathrm{b} & \mu \text { (total) } \\ \text { Isothiazole } & 1 \cdot 1 \pm 0 \cdot 1 & 2 \cdot 2 \pm 0 \cdot 1 & 2 \cdot 4_{4} \pm 0 \cdot 2 \\ \text { 1,2,4-Oxadiazole } & 1 \cdot 2 \pm 0 \cdot 2 & 0 \cdot 2 \pm 0 \cdot 2 & 1 \cdot 2 \pm 0 \cdot 3 \\ \text { Imidazole } & 3 \cdot 7 \pm 0 \cdot 3 & 0 \cdot 9 \pm 0 \cdot 3 & 3 \cdot 8 \pm 0 \cdot 4\end{array}$

Because in isothiazole the $A$ axis must approximately bisect the $\mathrm{C}-\mathrm{S}-\mathrm{N}$ angle, these data roughly define two possible lines of action of the total dipole in the molecule. The total moment agrees well with a recent determination from dielectric measurements ${ }^{8}$. For 1,2,4-oxadiazole the moment found is notably less than those of the other oxadiazoles. Unpublished molecular orbital calculations by Davies and Mackrodt show that this difference is theoretically predictable and give good agreement with the moment found here. The dipole moment of imidazole has been given rather scattered values from dielectric experiments ${ }^{8}$. Our result is close to the lower limit of the values obtained in solution and agrees closely with the moment calculated theoretically by Brown and Coller ${ }^{10}$. From the experimental $A$ and $B$ components of the moment in imidazole, the line of action of the moment is found to make an angle of approximately $13^{\circ}$ with the $A$ axis. This line of action can only agree well with that predicted by Brown and Coller ${ }^{10}$ if the $A$ axis intersects the $\mathrm{N}-\mathrm{C}$ bond joining positions 1 and 5 , but this evidence may not be strong enough to compel a preference for this orientation of the axes.

It is clear that much fuller information about the orientation of axes of inertia, lines of action of dipole moments and axes of quadrupole coupling tensors will follow from extended studies, in progress or planned, involving isotopic forms of these molecules. Refinement of the Stark measurements will require allowance for quadrupole splittings and, in the case of 1,2,4-oxadiazole and imidazole, for quartic Stark effect corrections ${ }^{6}$. Such studies promise exceptionally full data on both electron distributions and internuclear distances for comparison with the results of extensive theoretical treatments.

This research was sponsored, in part, by a grant from the Air Force Office of Scientific Research through the European Office of Aerospace Research (OAR), US Air Force. We thank Dr Ronald Slack for the gift of isothia. zole, Dr C. Moussebois for a sample of 1,2,4-oxadiazole and the Science Research Council for a research grant. Two of us (A. W. and J. H. G.) also thank the Council for research studentships.

\section{J. H. GRIFFIthS \\ A. WARDLEY Valerie E. Williams \\ N. L. OWEN \\ J. ShERIDAN}

Department of Chemistry,

University College of North Wales,

Bangor.

Received November 27, 1967.

${ }^{1}$ Bak, B., Christensen, D., Dixon, W. B., Nygaard, L., Rastrup-Andersen. .J., and Schottländer, M., J. Mol. Spectros., 9, 124 (1962).

- Bak, B., Christensen, D., Hansen, L., and Rastrup-Andersen, J., J. Chem. Phys, 24, 720 (1956).

${ }^{3}$ Saegebarth, E., and Cox, A. P., J. Chem. Phys., 43, 166 (1965).

Bak, B., Nielsen, J. T., Nielsen, O. F., Nygarard, L., Rastrup-Andersen, J., and Steiner, P. A., J. Mol. Spectros., 19, 458 (1966).

Bak, B., Christensen. D., Nygaard, L., and Rastrup-Andersen, J., J. Mol. Spertros., 9, $222(1962)$.

${ }^{6}$ Kirchhoff, W. H., J. Amer. Chem. Soc., 89, 1312 (1967).

'Martinez-Carrera. S., Acta. Cryst., 20, 783 (1966).

- Franchini, P. F., Trans. Farad. Soc., 63, 2610 (1967).

MeClellan, A. L., in Tables of Experimental Dipole Moments, 78 (Freeman, 1963).

${ }^{10}$ Brown, R. D., and Coller, B. A. W., Theor. Chim. Acta, 7, 259 (1967). 